\title{
10-Day structured initiation protocol from multiple daily injection to hybrid closed-loop system in children and adolescents with type 1 diabetes
}

\author{
Goran Petrovski ${ }^{1}$ (D) Fawziya Al Khalaf ${ }^{1} \cdot$ Judith Campbell $^{1} \cdot$ Hannah Fisher $^{1} \cdot$ Fareeda Umer $^{1} \cdot$ Khalid Hussain $^{1}$
}

Received: 28 November 2019 / Accepted: 27 December 2019 / Published online: 17 January 2020

(c) The Author(s) 2020

\begin{abstract}
Aim The aim of this study was to evaluate the 10-day initiation protocol for MiniMed 670G hybrid closed-loop (HCL) system in individuals with type 1 diabetes on multiple daily injection (MDI) in achieving desirable glycemic control.

Methods An open-label single-arm, single-center, clinical investigation in children aged 7-18 years on MDI following a structured protocol: 2 days, HCL system assessment; 5 days, HCL system training (2-h sessions on 5 consecutive days with groups of 3-5 participants and families); 3 days, Manual Mode use of HCL system; 84 days, Auto Mode use of the HCL system, cumulating in 10 days from MDI to Auto Mode activation.

Results A total of 30 children (age 10.24 \pm 2.6 years) were enrolled in the study, and all completed the planned 84 days on Auto Mode. The participants used the sensor for a median of $92 \%$ of the time and spent a median of $89 \%$ in Auto Mode. The mean $\mathrm{HbA} 1 \mathrm{c}$ decreased from $8.2 \pm 1.4 \%(66 \pm 15.3 \mathrm{mmol} / \mathrm{mol})$ at baseline to $6.7 \pm 0.5 \%(50 \pm 5.5 \mathrm{mmol} / \mathrm{mol})$ at the end of the study $(p=0.017)$. Time in range $(70-180 \mathrm{mg} / \mathrm{dL})$ increased from $46.9 \pm 18.5 \%$ at baseline to $75.6 \pm 6.9 \%$ in Auto Mode $(p<0.001)$. This was achieved while spending $2.8 \%$ of the time below $70 \mathrm{mg} / \mathrm{dL}$ and without any severe hypoglycemia or DKA.

Conclusion Children and adolescents with type 1 diabetes on MDI therapy can successfully initiate the HCL system, using a concise structured 10-day protocol.
\end{abstract}

Keywords Hybrid closed-loop system · Multiple daily injection · Protocol · Type 1 diabetes

\section{Introduction}

Improving glycemic control in individuals with type 1 diabetes without increasing risk of hypoglycemia is a challenge for both individuals and health providers. Intensive insulin treatment in combination with regular self-monitoring of blood glucose (SMBG) is the standard of care for individuals with type 1 diabetes. Nevertheless, the majority of individuals fail to achieve optimal glycemic control with $\mathrm{HbA1c}$ below 7\% (53 mmol/mol) [1]. Monitoring glucose levels

Managed by Massimo Porta.

Goran Petrovski

gpetrovski@sidra.org

1 Division of Endocrinology and Diabetes, Department of Pediatric Medicine, Sidra Medicine, HB 6E 219, Al Luqta Street, Education City North Campus, PO Box 26999, Doha, Qatar from the interstitial fluid, either continuously with real-time continuous glucose monitoring (CGM) or intermittently, by scanned continuous glucose monitoring (isCGM), demonstrated better diabetes management for children and adolescents with diabetes [2].

Recent technological advances in diabetes treatment have integrated continuous subcutaneous insulin delivery (CSII) with CGM, where insulin delivery can be automated by sensor glucose (SG)-driven algorithms. Insulin delivery suspension when reaching a low glucose level $[3,4]$ or in prediction of a low glucose level $[5,6]$ has demonstrated significant reduction in hypoglycemia exposure.

Further development in technology of integrated closedloop systems provides algorithm-derived automated adjustment of insulin delivery to address both hypoglycemia and hyperglycemia [7-11].

The first approved hybrid closed-loop (HCL) system [12] is the MiniMed 670G system (Medtronic Diabetes, Northridge, CA, USA) for children above 7 years old [13], 
adolescents and adults with type 1 diabetes. The system [14] uses an algorithm to adjust basal insulin delivery automatically every $5 \mathrm{~min}$ based on SG values $[15,16]$. Several studies have shown improved HbAlc, time in target range, and SG variability in children [17], adolescents and adults [18, 19] with type 1 diabetes.

Studies in MiniMed 670G system [17, 19] included participants experienced with using CSII therapy, assuming that the success of HCL systems depends on prior use of technology. There is no evidence of the glycemic control achieved by patients on multiple daily injection (MDI) transitioning to the HCL system. Although a recent publication suggested a training and education program for HCL system in patients naïve to insulin pump therapy [20], there is no standardized protocol to initiate HCL system in individuals on MDI.

The objective of this study was to evaluate whether the use of a 10-day structured initiation protocol for MiniMed 670G HCL system in individuals with type 1 diabetes on MDI therapy will provide similar outcomes to the MiniMed 670G pivotal studies, that included individuals on pumps therapy and not patients on MDI, as the latter are the majority of individuals with type 1 diabetes [17, 19].

\section{Methods}

This open-label, non-randomized, single-arm, single-center, clinical investigation study was conducted at Sidra Medicine in Doha, Qatar, and enrolled individuals aged 7-18 years with type 1 diabetes $>1$ year, on MDI with SMBG, with or without RT-CGM or isCGM, with no prior pump experience, and with an $\mathrm{HbA} 1 \mathrm{c}$ level $<12.5 \%$. Individuals were recruited at regular clinic visits, following the clinical pathway for therapy [21]. This clinical assessment emphasizes the following individual aspects: frequency of SMBG controls per day, knowledge of carbohydrate counting, insulin dose adjustments based on carbohydrate intake and blood glucose level, the ability to identify changes in insulin requirements due to physical activity and sick days, ability to recognize, troubleshoot and manage hyperglycemia and hypoglycemia. Individuals with self-funding capability and those with insurance coverage were recruited in the study.

The initiation protocol consisted of four main stages: HCL system compatibility assessment, HCL system training, Manual Mode and Auto Mode stages.

\section{Step 1: HCL system compatibility assessment}

Interested individuals attended two introduction sessions of $1 \mathrm{~h}$ (groups of 8-12), where the MiniMed 670G system was described. Individuals' responsibilities (bolusing before meal, calibrating the system 3-4 times per day, responding to alerts and alarms, downloading pump data from home) and expectations (improvements in glycemic control, less glucose variability, hypoglycemia minimization) were discussed.

\section{Step 2: HCL system training}

Three-to-five individuals and their parents/guardians attended group training program. The program included five sessions of $2 \mathrm{~h}$ on five consecutive days: Day $1-$ pump buttons and menus, understanding CGM graph, education in sensor calibration and sensor insertion; Day 2-basic training of the different pump modes: Manual Mode, bolus wizard use, basal rates, Auto Mode, introducing the concepts of safe basal; Day 3-practical training on infusion set and reservoir change, Medtronic CareLink Software downloads and creation of personal accounts; Day 4hypoglycemia, hyperglycemia, exercise and travel management, guidance on the use of temporary target; Day 5-explaining Auto Mode readiness screen, instructing on meal bolus (timing and carb counting refresher), correction bolus, system exits and final evaluation of participants' ability to handle the HCL system.

Each session was provided by two educators from $12 \mathrm{pm}$ to $2 \mathrm{pm}$. CGM was initiated on the first day of the training, for education and observational purposes and for baseline data collection (no insulin delivery by the pump). Timing of the long acting insulin injection was moved $2 \mathrm{~h}$ ahead each day during training sessions, to reach $12 \mathrm{pm}$ the day before the HCL system was initiated in Manual Mode, to avoid the use of temporary basal at insulin pump initiation.

\section{Step 3: Manual mode}

Participants initiated the use of the HCL system in Manual Mode with suspend before low feature for $72 \mathrm{~h}$ to allow the algorithm to collect insulin utilization and CGM data to establish personalized Auto Mode initiation parameters. Sidra's validated protocol for SAP initiation, as previously described [22], with review of 1-week CGM data (step 2) was used: In short, the protocol inputs the current insulin program (MDI) and calculates a $10-20 \%$ reduction in total daily dose, with a $40 / 60$ basal/bolus distribution in four or five basal rates. Insulin-to-carbohydrate ratio (ICR) settings utilize the formula of 300-450/total daily dose (TDD) and the formula of $90-110 / \mathrm{TDD}(\mathrm{mmol} / \mathrm{L})$ with two CF settings; the nighttime CF factor is set $10-20 \%$ higher than the daytime CF. Active insulin is set time $(3 \mathrm{~h})$; suspend before low feature is turned on with a threshold of $3.0-3.8 \mathrm{mmol} / \mathrm{L}$ $(55-70 \mathrm{mg} / \mathrm{dL})$, and glucose target ranges from 5.0 to $7.2 \mathrm{mmol} / \mathrm{L}(90-130 \mathrm{mg} / \mathrm{dL})$. 


\section{Step 4: Auto mode}

The Auto Mode feature of the HCL system was activated $72 \mathrm{~h}$ after Manual Mode initiation and used continuously for 84 days. Follow-up visits were scheduled as follows: in clinic on days 3, 7 and 84; and phone call visits on days 14, 28 and 56 after enabling Auto Mode. Participants uploaded data from the pump to CareLink Personal software (Medtronic, Northridge, CA, USA) every 2 weeks.

HbA1c was obtained using point of care DCA Vantage Analyzer (Siemens, Erlangen, Germany) at baseline and at the end of the study.

The study was approved by local and National Ethics Committee in Qatar, and all participants and their guardians signed an informed consent document.

\section{Statistical analysis}

Analysis was performed for the entire study population. Sub-analysis for prior CGM users versus users without prior CGM was predefined. Prior CGM use was defined as a use of Dexcom G5 (Dexcom, San Diego, CA, USA), Guardian Connect (Medtronic, Northridge, CA, USA) or Freestyle libre (Abbott Diabetes Care, Berkshire, UK) at baseline, in combination with SMBG and MDI.

Insulin dosing information and CGM data were collected from CareLink Therapy Management Software during the study. All data are presented as mean \pm SD, median, interquartile or as a percentage. The paired student $t$ test or paired Wilcoxon test, in case of non-normality, was used in the study. A value of 0.05 was considered statistically significant. Statistical analyses were performed using Statistica 12 (Stat Soft, Tulsa, USA).

\section{Results}

Thirty-eight individuals met the criteria for HCL system use, of these 30 children (age 10.24 \pm 2.6 years) consented to initiate the system and were enrolled in the study. The 10-day initiation protocol was implemented in 30 participants, and they all completed the planned 84 days on Auto Mode. Baseline characteristics are shown in Table 1.

After activating Auto Mode, the participants used the sensor for a median of $92 \%$ of the time and spent a median of $89 \%$ in Auto Mode at the end of the study.

$\mathrm{HbA} 1 \mathrm{c}$ decreased from $8.2 \pm 1.4 \%(66 \pm 15.3 \mathrm{mmol} / \mathrm{mol})$ at baseline, to $6.7 \pm 0.5 \%(50 \pm 5.5 \mathrm{mmol} / \mathrm{mol})$ at the end of the study $(p=0.017)$. Time in range (TIR) $(70-180 \mathrm{mg} / \mathrm{dL})$ increased from $46.9 \pm 18.5 \%$ at baseline to $74.8 \pm 7.1 \%$ in Auto Mode $(p<0.001)$. Time below range did not change, while time above range decreased significantly (Table 2). Mean SG decreased from $193 \pm 41$ to $142 \pm 12 \mathrm{mg} / \mathrm{dL}$
Table 1 Study participant's characteristics at baseline

\begin{tabular}{ll}
\hline & $\begin{array}{l}\text { Participants } \\
N=30\end{array}$ \\
\hline Age, years & $10.24 \pm 2.6$ \\
Male, $n(\%)$ & $15(50 \%)$ \\
Female, $n(\%)$ & $15(50 \%)$ \\
Weight, kg & $38.2 \pm 12.5$ \\
BMI, kg/m ${ }^{2}$ & $18.6 \pm 3.4$ \\
BMI, $z$-score & $0.2 \pm 0.9$ \\
Duration of diabetes, years & $2.8 \pm 1.7$ \\
TDD, U/(kg/day) & $0.8 \pm 0.3$ \\
HbA1c, $\%$ & $8.2 \pm 1.4$ \\
HbA1c, mmol/mol & $66 \pm 15.3$ \\
Sensor use, $n(\%)$ & \\
RT-CGM & $6(20 \%)$ \\
isCGM & $10(33 \%)$ \\
No sensor & $14(47 \%)$ \\
\hline
\end{tabular}

All values are shown as mean $\pm \mathrm{SD}$, except for gender and sensor use $B M I$ body mass index, $T D D$ total daily dose of insulin, $S D$ standard deviation, $R T$-CGM real-time continuous glucose monitoring (Dexcom G5/Guardian Connect), isCGM intermittent continuous glucose monitoring (freestyle libre)

$(p=0.001)$. TDD and the percentage of basal insulin delivered increased by a mean of $0.1 \mathrm{Unit} / \mathrm{kg}(p=0.02)$ during the study, compared to the baseline (Table 2).

\section{Time in ranges evolution over time}

Comparing different study periods, TIR (70-180 mg/dL) increased from MDI + CGM to Manual Mode $(p=0.010)$; Manual Mode to Auto Mode of 3 days $(p=0.003)$; Auto Mode of 3-28 days $(p=0.006)$; Auto Mode of 28-56 days $(p=0.698)$ and Auto Mode of 57-86 days $(p=0.147)$, as shown in Fig. 1. Time above range $(>181 \mathrm{mg} / \mathrm{dL})$ decreased from MDI + CGM to Auto Mode of 57-84 days during the study $(p=0.021)$.

\section{HCL system usability}

The total number of Auto Mode exits during the 3 months study phase was $5.1 \pm 1.2$ events per week, and significantly decreased between the first 2 weeks and the third month after enabling Auto Mode, from $8.4 \pm 1.8$ to $4.2 \pm 0.9$ events per week, respectively ( $p=0.016$ ) (Table 3$)$. The mean number of daily calibrations also significantly decreased from $4.3 \pm 1.2$ to $3.4 \pm 0.9(p=0.010)$. Infusion set and reservoir were changed on a regular basis every 2-3 days. Number of meals and carbohydrate intake did not differ from the beginning to the end of the study. ICR during initial Auto Mode period was made more aggressive regardless of the meal period compared with baseline and decreased from a 
Table 2 Glucose control, $\mathrm{HbA} 1 \mathrm{c}$, insulin delivered during baseline and study phase

\begin{tabular}{llll}
\hline & Baseline & Study & $p$ \\
\hline HbA1c $(\%)$ & $8.2 \pm 1.4(8.3,7.1-9.2)$ & $6.7 \pm 0.5(6.7,6.2-6.9)$ & 0.017 \\
HbA1c $(\mathrm{mmol} / \mathrm{mol})$ & $66 \pm 15.3(67,54-77)$ & $50 \pm 5.5(50,44-52)$ & 0.017 \\
Sensor glucose $(\mathrm{mg} / \mathrm{dL})$ & $193 \pm 41(191,158-227)$ & $142 \pm 12(141,132-148)$ & 0.001 \\
Percent of sensor glucose values in range & & \\
$\leq 50 \mathrm{mg} / \mathrm{dL}$ & $0.4 \pm 0.7(0.2,0.1-0.6)$ & $0.3 \pm 0.5(0.2,0.1-0.5)$ & 0.330 \\
$51-70 \mathrm{mg} / \mathrm{dL}$ & $2.7 \pm 3.7(2.5,1.2-3.4)$ & $2.5 \pm 1.8(2.4,1.2-2.2)$ & 0.507 \\
$71-180 \mathrm{mg} / \mathrm{dL}$ & $46.9 \pm 18.5(46.5,33-61)$ & $74.8 \pm 7.1(75.1,71.0-82.7)$ & 0.001 \\
$181-250 \mathrm{mg} / \mathrm{dL}$ & $25.8 \pm 8.8(25.2,22.1-32.4)$ & $16.9 \pm 5.7(16.8,12.1-20.7)$ & 0.001 \\
$\geq 251 \mathrm{mg} / \mathrm{dL}$ & $24.2 \pm 16.9(24.3,9.2-38.4)$ & $5.5 \pm 3.6(4.9,2.2-8.2)$ & 0.001 \\
TDD, U/(kg/day) & $0.8 \pm 0.3(0.8,0.6-1.0)$ & $0.9 \pm 0.2(0.8,0.7-0.9)$ & 0.020 \\
Basal insulin, as \% of TDD & $36.5 \pm 7.2(37.8,30,3-40.2)$ & $42.2 \pm 6.7(42.0,38.4-46.3)$ & 0.037 \\
Weight $(\mathrm{kg})$ & $38.3 \pm 12.5(38.6,26.5-48.4)$ & $39.4 \pm 8.9(38.9,27.5-49.1)$ & 0.636 \\
\hline
\end{tabular}

Baseline consisted of one week on MDI with CGM; study phase consisted of 12 weeks on Auto Mode. All values are shown as mean $\pm \mathrm{SD}$ (median, interquartile range)

$M D I$ multiple daily injections, $S G$, sensor glucose, $T D D$ total daily dose of insulin

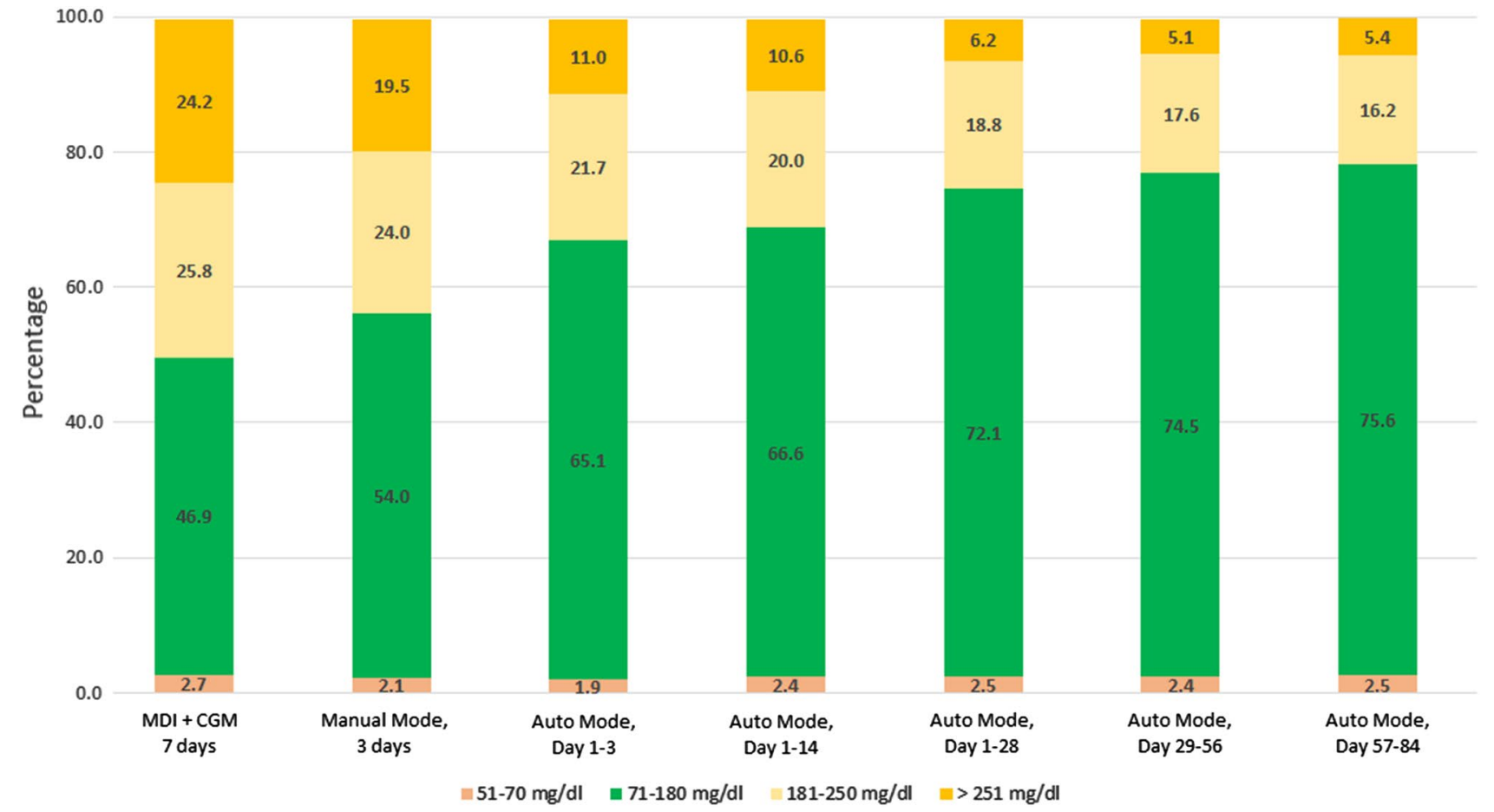

Fig. 1 Time in ranges at baseline, during Manual Mode and Auto Mode periods. Values are shown as percentage spent in ranges during the interval. $M D I$ multiple daily injections, $C G M$ continuous glucose

median of 15 at the beginning to 12 at the end of the study $(p=0.002)$.

\section{Prior CGM versus no prior CGM users}

$\mathrm{HbA} 1 \mathrm{c}$ decreased in both prior CGM and no prior CGM users, from $8.1 \pm 1.6 \%(65 \pm 17.5 \mathrm{mmol} / \mathrm{mol})$ to $6.7 \pm 1.1$ monitoring. Glucose values $<50 \mathrm{mg} / \mathrm{dL}$ are not shown on the graph: $0.3 \%$ in Manual Mode and Auto Mode period day $57-84,0.4 \%$ in all other periods

$(50 \pm 12 \mathrm{mmol} / \mathrm{mol})(p=0.021)$, and from $8.2 \pm 0.9 \%$ $(66 \pm 9.8 \mathrm{mmol} / \mathrm{mol})$ to $6.6 \pm 1.6 \%(49 \pm 17.5 \mathrm{mmol} / \mathrm{mol})$ $(p=0.019)$, respectively. TIR improved from baseline to end of the study in both groups, reaching $75.2 \pm 5.9$ and $75.9 \pm 7.3$ during the third month in Auto Mode, respectively. No significant difference in HbA1c or TIR was found between these groups at the end of the study. We did not find 
Table 3 HCL system characteristics during the Auto Mode period

\begin{tabular}{|c|c|c|c|}
\hline & First 2 weeks of Auto Mode & Third month in Auto Mode & $p$ \\
\hline Sensor wear, \% & $89.8 \pm 7.5(92,90-94)$ & $92.4 \pm 6.2(94,86-96)$ & 0.038 \\
\hline Auto Mode usage, $\%$ & $84.8 \pm 11.9(87,77-97)$ & $89.8 \pm 7.4(90,84.5-96.5)$ & 0.787 \\
\hline Calibration, $n$ per day & $4.3 \pm 1.2(3.8,3.2-4.9)$ & $3.4 \pm 0.9(3.4,2.7-4.3)$ & 0.010 \\
\hline Set change, $n$ of days & $2.5 \pm 0.7(3.0,2-3)$ & $2.8 \pm 0.6(3.0,2.4-3.1)$ & 0.269 \\
\hline Res change, $n$ of days & $2.9 \pm 1.3(2.2-2.3)$ & $2.8 \pm 0.6(2.1-3.5)$ & 0.315 \\
\hline Meals, $n$ per day & $4.4 \pm 1.2(4.4,3.8-5.2)$ & $4.6 \pm 1.2(4.4,3.8-5.0)$ & 0.331 \\
\hline Carbs, gram per day & $213 \pm 50(205,173-254)$ & $219 \pm 48(221,190-242)$ & 0.704 \\
\hline ICR, gram & $16.2 \pm 5.7(15,12-20)$ & $12.8 \pm 4.8(12,10-16)$ & 0.002 \\
\hline Active insulin time, $\mathrm{h}$ & $3.6 \pm 0.4(3.3,3.3-4.0)$ & $3.5 \pm 0.4(3.5,3-4)$ & 0.155 \\
\hline \multicolumn{4}{|c|}{ Auto Mode exits per patient per week } \\
\hline Total number ${ }^{\mathrm{a}}$ & $8.4 \pm 1.8$ & $4.2 \pm 0.9$ & 0.016 \\
\hline No calibration $^{\mathrm{a}}$ & $3.3 \pm 2.6$ & $1.3 \pm 1.5$ & 0.004 \\
\hline High $S^{a}$ & $2.8 \pm 1.7$ & $1.2 \pm 1.8$ & 0.024 \\
\hline Insulin max delivery ${ }^{\mathrm{a}}$ & $1.1 \pm 0.9$ & $0.5 \pm 0.3$ & 0.005 \\
\hline
\end{tabular}

Values are shown as mean $\pm \mathrm{SD}$, median and interquartile ranges

$n$ number, Res reservoir, Carbs carbohydrates, ICR insulin-to-carb ratio, $S G$ sensor glucose, Max maximum. Only the top three Auto Mode exits reasons are shown

${ }^{\text {a}}$ Values shown as mean and SD significant difference in sensor wear, Auto Mode usage and numbers of calibrations between prior CGM and no prior CGM users at the end of the study.

\section{Safety}

There was no event of severe hypoglycemia, diabetic ketoacidosis (DKA) or hospital admission during the study.

\section{Discussion}

This study describes a structured initiation protocol of MiniMed 670G HCL system in individuals with type 1 diabetes on MDI, and its impact on glycemic control and safety for 3 months following Auto Mode activation. Totally, 79\% of eligible candidates consented to the study and no attrition was observed during the 3-month follow-up. The patients will be monitored to determine long-term persistence. The high individual engagement demonstrated in our study was exemplified by high sensor usage and percent time in Auto Mode. Median sensor use was $92 \%$ of the time, which is similar to previous reports of $90.9 \%$ in children [17], of $88.6 \%$ in adolescent and of $93.1 \%$ in adults [19]. They spent a median of $89 \%$ of the time in Auto Mode, which is significantly higher compared to previous reports of medians of $80.6 \%$ in children [17], $75.8 \%$ in adolescents and $88.0 \%$ in adults [19] and $84.8 \%$ in another study [20]. Participants' high engagement and no attrition can be an indicator of their motivation and satisfaction with the HCL system in improving their glycemic control.
The reduction in $\mathrm{HbA} 1 \mathrm{c}$, by a mean of $1.5 \%(16 \mathrm{mmol} /$ $\mathrm{mol})$, and in the SG, by a mean of $53 \mathrm{mg} / \mathrm{dL}(2.9 \mathrm{mmol} / \mathrm{mol})$, observed in our study is greater than that previously reported in children [17] and adolescents, and similar to adults [19]. Totally, $70 \%$ of the participants reached $\mathrm{HbA} 1 \mathrm{c}<7.0 \%$ $(53 \mathrm{mmol} / \mathrm{mol})$ at the end of the study, which is the target established by the ADA and ISPAD guidelines for glycemic control in children [1,2]. This is also significantly superior to what was observed in the previous MiniMed 670G pivotal trials.

The time spent within, below and above the target glycemic ranges has been defined as glycemic goals, beyond HbA1c [23, 24]. In our study, the median TIR (70-180 mg/ $\mathrm{dL}$ ) achieved in Auto Mode was $75.2 \%$, which is similar to the previously reported medians of $73.5 \%$ [18] and $74.9 \%$ [19] in adults, but significantly higher than a median of $68.8 \%$ in adolescents [19] and $64.6 \%$ in children [17]. Time in range, time below range, and time above range observed in our study all achieved the desired clinical targets for CGM data interpretation, recently published by International Consensus on Time in Range [25]. These superior clinical outcomes, compared to the ones reported in previous studies of the MiniMed 670G system, which included experienced insulin pump users, were most probably driven by the high sensor and Auto Mode use. It is therefore possible that our specific initiation protocol, the parental/guardian involvement and supervision of the children, as well as the support and follow-up by the diabetes team, lead to the higher percentage of time spent in Auto Mode.

The significant TIR improvement was observed after only 3 days in Auto Mode, which indicates the effectiveness of 
our 10-days structured initiation protocol, as well as the rapid adaptation of the HCL system to the specific needs of the individual. After 3 days, TIR continuously improved over time until reaching a plateau after 2 months.

Our initiation HCL protocol for individuals on MDI allows to enable the Auto Mode feature in a 10-day period, which is significantly shorter compared to the HCL protocol group training for patient naïve to insulin pump therapy [20], where Auto Mode feature was enabled after 3-4 weeks from initial training. In the pivotal $670 \mathrm{G}$ studies in children [17], adolescent and adults [19], participants spent about 3 weeks in Manual Mode followed by 1-2 weeks of pump training, before Auto Mode was enabled. In our study, the short period of pump training and Manual Mode did not impact the glycemic control and safety of the participants; on the contrary, the superior clinical outcomes that were achieved in $\mathrm{HbA1c}(6.7 \pm 0.5 \%(50 \pm 5.5 \mathrm{mmol} / \mathrm{mol}))$ and in median TIR (70-180 mg/dL) of $75.2 \%$ undermine the concept that closed-loop systems use require prior experience with diabetes-related technology to be effective and safe.

Additional insight from our experience is that modifying ICR by increasing the meal bolus dose by almost $20 \%$ during the first month of Auto Mode use is necessary when initiating individuals on HCL system from MDI regimens. Previous studies of HCL system reported similar findings [26]. This is dependent on the specifics of the algorithm [27-29] and may not be applicable for all HCL systems [30].

Both TDD and the percentage of daily basal insulin delivered slightly increased, which is similar to previously reported studies $[17,19]$. Automated basal insulin delivery and ICR modifications, driving increase in meal bolus, effectively distributed the insulin delivery according to patients' individual requirements, resulting in better control with minimal increase in total insulin dosages.

Similar results were achieved by participants with and without prior use of CGM, reinstating that the lack of prior experience with diabetes-related technology does not hinder the outcome with the MiniMed 670G system [31].

Auto Mode exits averaged 5.1 \pm 1.2 per week in the study period, which is similar to previously published results of 5-6 exits [26] and 5.8 \pm 1.6 exits [17], again attesting to the fact that prior insulin pump and/or CGM experience is not necessary for proper handling of the HCL system.

The main reasons for exiting Auto Mode were no calibration, high SG and maximum insulin delivery. The number of Auto Mode exits decreased from $8.4 \pm 1.8$ to $4.2 \pm 0.9$ events per week $(p=0.016)$, between the first 2 weeks and the third month on Auto Mode, respectively. In the same periods, the number of sensor calibrations decreased from $4.3 \pm 1.2$ to $3.4 \pm 0.9$ per day. This can indicate that the participants became more comfortable using the system over time, which is supported by the increased time spent in Auto Mode and the increased TIR achieved over time.
Importantly, the improved clinical outcomes observed in our study were achieved in a safe manner, with no events of DKA, or severe hypoglycemia, and with no hospital admission, similar to the MiniMed 670G pivotal trials.

There are several limitations of our study: absence of a control group, relatively short observation period (3 months) and different time periods in the evaluation of glycemic control. Additional limitation is that the protocol assessed only the minimal $72 \mathrm{~h}$ period in Manual Mode before turning Auto Mode on. Longer periods in Manual Mode might have had additional benefits. However, the objective of this study was to evaluate the protocol to initiate HCL system in individuals on MDI therapy, in comparison with the clinical outcomes reported in previous studies of the same HCL system.

\section{Conclusion}

Children and adolescents with type 1 diabetes on MDI therapy can successfully initiate the HCL system, using a concise structured 10-day protocol, achieving better outcomes than in previous studies, where participants had previous experience with diabetes-related technology and longer initiation process. Further investigation on more varied population and ages should be performed to confirm these findings.

Acknowledgements Open Access funding provided by the Qatar National Library. The authors thank the participants and their families for their collaboration during the study and acknowledge the contributions and the efforts of administration and research staff at Sidra Medicine for data management and analyses. G.P. researched the data and wrote the manuscript. F.A., J.C. and K.H. contributed to the discussion and reviewed and edited the manuscript. J.C., H.F. and F.U. contributed to the patients' education and follow-up. G.P. is the guarantor of this work and, as such, had full access to all the data in the study and takes responsibility for the integrity of the data and the accuracy of the data analysis.

Funding This study (ClinicalTrials.gov NCT03755479) was funded by Sidra Medicine, Doha.

\section{Compliance with ethical standards}

Conflict of interest The authors declare that they have no conflict of interest.

Ethical approval This study was approved by local (Sidra Medicine) and National Ethics Committee in Qatar and all participants and their guardians signed an informed consent document.

Human and animal rights All procedures followed were in accordance with the ethical standards of the responsible committee on human experimentation (institutional and national) and with the Helsinki Declaration of 1975, as revised in 2005 .

Informed consent Informed consent was obtained from all patients for being included in the study. 
Open Access This article is licensed under a Creative Commons Attribution 4.0 International License, which permits use, sharing, adaptation, distribution and reproduction in any medium or format, as long as you give appropriate credit to the original author(s) and the source, provide a link to the Creative Commons licence, and indicate if changes were made. The images or other third party material in this article are included in the article's Creative Commons licence, unless indicated otherwise in a credit line to the material. If material is not included in the article's Creative Commons licence and your intended use is not permitted by statutory regulation or exceeds the permitted use, you will need to obtain permission directly from the copyright holder. To view a copy of this licence, visit http://creativecommons.org/licenses/by/4.0/.

\section{References}

1. American Diabetes Association (2019) Glycemic targets: standards of medical care in diabetes-2019. Diabetes Care 42(11):S61-S70

2. DiMeglio LA, Acerini CL, Codner E et al (2018) ISPAD clinical practice consensus guidelines 2018: glycemic control targets and glucose monitoring for children, adolescents, and young adults with diabetes. Pediatr Diabetes 19(S27):105-114

3. Ly TT, Nicholas JA, Retterath A et al (2013) Effect of sensoraugmented insulin pump therapy and automated insulin suspension vs standard insulin pump therapy on hypoglycaemia in patients with type 1 diabetes. A randomized clinical trial. JAMA 310(12):1240-1247

4. Bergenstal RM, Klonoff DC, Garg SK, for the ASPIRE In-Home Study Group et al (2013) Threshold-based insulin pump interruption for reduction of hypoglycemia. N Engl J Med 369:224-232

5. Bosi E, Choudhary P, de Valk HW et al (2019) Efficacy and safety of suspend-before-low insulin pump technology in hypoglycaemia-prone adults with type 1 diabetes (SMILE): an openlabel randomised controlled trial. Lancet Diabetes Endocrinol 7(6):462-472

6. Battelino T, Nimri R, Dovc K, Phillip M, Bratina N (2017) Prevention of hypoglycemia with predictive low glucose insulin suspension in children with type 1 diabetes: a randomized controlled trial. Diabetes Care 40:764-770

7. Thabit H, Hovorka R (2016) Coming of age: the artificial pancreas for type 1 diabetes. Diabetologia 59:1795-1805

8. Kropff J, DeVries JH (2016) Continuous glucose monitoring, future products, and update on worldwide artificial pancreas projects. Diabetes Technol Ther 18(S2):S253-S263

9. Shah VN, Shoskes A, Tawfik B, Garg SK (2014) Closed loop system in the management of diabetes: past, present, and future. Diabetes Technol Ther 16:477-490

10. Biester T, Nir J, Remus K et al (2018) DREAM5: an open-label, randomized, cross-over study to evaluate the safety and efficacy of day and night closed-loop control by comparing the MD-Logic automated insulin delivery system to sensor augmented pump therapy in patients with type 1 diabetes at home. Diabetes Obes Metab 26:1-7

11. Nimri R, Bratina N, Kordonouri O et al (2017) MD-Logic overnight type 1 diabetes control in home settings: multicenter, multinational, single blind, randomized trial. Diabetes Obes Metab 19:553-561

12. U.S. Food and Drug Administration (2019) FDA approves first automated insulin delivery device for type 1 diabetes. www.fda.gov/ news-events/press-announcements/fda-approves-first-automatedinsulin-delivery-device-type-1-diabetes. Accessed 21 Mar 2019

13. U.S. Food and Drug Administration (2019) FDA approves automated insulin delivery and monitoring system for use in younger pediatric patients. www.fda.gov/news-events/press-announceme nts/fda-approves-automated-insulin-delivery-and-monitoring -system-use-younger-pediatric-patients. Accessed 21 July 2019

14. U.S. Food and Drug Administration (2019) FDA summary of safety and effectiveness data; PMA P160017. www.accessdata .fda.gov/cdrh_docs/pdf16/P160017B.pdf. Accessed 18 Apr 2019

15. Weaver KW, Hirsch IB (2018) The hybrid closed-loop system: evolution and practical applications. Diabetes Technol Ther 20(S2):S216-S223

16. Trevitt S, Simpson S, Wood A (2016) Artificial pancreas device systems for the closed-loop control of type 1 diabetes: what systems are in development. J Diabetes Sci Technol 10:714-723

17. Forlenza GP, Pinhas-Hamiel O, Liljenquist DR et al (2019) Safety evaluation of the MiniMed 670G system in children 7-13 years of age with type 1 diabetes. Diabetes Technol Ther 21(1):11-19

18. Bergenstal RM, Garg S, Weinzimer SA et al (2016) Safety of a hybrid closed-loop insulin delivery system in patients with type 1 diabetes. JAMA 316:1407-1408

19. Garg SK, Weinzimer SA, Tamborlane WV et al (2017) Glucose outcomes with the in-home use of a hybrid closed-loop insulin delivery system in adolescents and adults with type 1 diabetes. Diabetes Technol Ther 19:155-163

20. Aleppo G, Webb KM (2018) Integrated insulin pump and continuous glucose monitoring technology in diabetes care today: a perspective of real-life experience with the MiniMed ${ }^{\mathrm{TM}}$ 670G hybrid closed-loop system. Endocr Pract 24(7):684-692

21. Petrovski G, Al Khalaf F, Hussain K et al (2018) Continuous subcutaneous insulin infusion characteristics in type 1 diabetes children and adolescents in Qatar. Diabetes Ther 9(5):2091-2098

22. Petrovski G, Zivkovic M, Stratrova SS et al (2017) Type 1 diabetes and long-term continuous subcutaneous insulin infusion therapy: a 10-year experience from Macedonia. Diabetes Technol Ther 19(12):739-743

23. Rodbard D (2017) Continuous glucose monitoring: a review of recent studies demonstrating improved glycemic outcomes. Diabetes Technol Ther 19(S3):S25-S37

24. Wright LA, Hirsch IB (2017) Metrics beyond hemoglobin A1C in diabetes management: time in range, hypoglycemia, and other parameters. Diabetes Technol Ther 19(S2):S16-S26

25. Battelino T, Danne T, Bergenstal R et al (2019) Clinical targets for continuous glucose monitoring data interpretation: recommendations from the international consensus on time in range. Diabetes Care. https://doi.org/10.2337/dci19-0028

26. Messer LH, Forlenza GP, Sherr JL et al (2018) Optimizing hybrid closed-loop therapy in adolescents and emerging adults using the MiniMed 670G system. Diabetes Care 41(4):789-796

27. Messer LH, Forlenza GP, Wadwa RP et al (2018) The dawn of automated insulin delivery: a new clinical framework to conceptualize insulin administration. Pediatr Diabetes 19:14-17

28. Tauschmann M, Allen JM, Wilinska ME et al (2016) Day-andnight hybrid closed-loop insulin delivery in adolescents with type 1 diabetes: a free-living, randomized clinical trial. Diabetes Care 39:1168-1174

29. Doyle FJ 3rd, Huyett LM, Lee JB et al (2014) Closed-loop artificial pancreas systems: engineering the algorithms. Diabetes Care 37:1191-1197

30. Dassau E, Pinsker JE, Kudva YC et al (2017) Twelve-week 24/7 ambulatory artificial pancreas with weekly adaptation of insulin delivery settings: effect on hemoglobin A1c and hypoglycemia. Diabetes Care 40:1719-1726

31. Cordero TL, Garg SK, Brazg RL et al (2017) The effect of prior continuous glucose monitoring use on glycemic outcomes in the pivotal trial of the MiniMed ${ }^{\mathrm{TM}}$ 670G hybrid closed-loop system. Diabetes Technol Ther 16:338-347

Publisher's Note Springer Nature remains neutral with regard to jurisdictional claims in published maps and institutional affiliations. 